\title{
Growing Polymer Vesicles Generated by Polymerization Induced Self-Assembly Coupled With a Living Chemical Reactor
}

\author{
Zhihui Lu ${ }^{1 *}$ and Jinshan Guo ${ }^{2}$ \\ ${ }^{1}$ Department of Histology and Embryology, School of Basic Medical Sciences, Southern Medical University, Guangzhou, \\ China, ${ }^{2}$ Department of Earth and Planetary Science and Origin of Life Initiative, Harvard University, Cambridge, MA, \\ United States
}

OPEN ACCESS

Edited by:

Jianxun Ding,

Changchun Institute of Applied

Chemistry (CAS), China

Reviewed by:

Xianming Dai,

The University of Texas at Dallas,

United States

Yuandu $\mathrm{Hu}$,

Merck KGaA, Germany

Jun Yue,

Sun Yat-sen University, China

*Correspondence:

Zhihui Lu

Iuzhihui107@sohu.com

Specialty section:

This article was submitted to

Biomaterials,

a section of the journal

Frontiers in Bioengineering and

Biotechnology

Received: 14 July 2020

Accepted: 04 August 2020

Published: 01 September 2020

Citation:

Lu Z and Guo J (2020) Growing

Polymer Vesicles Generated by

Polymerization Induced Self-Assembly

Coupled With a Living Chemical

Reactor.

Front. Bioeng. Biotechnol. 8:1018.

doi: 10.3389/fbioe.2020.01018
Chemical oscillatory reactions have attracted intensive attention due to their autonomous, continuous, and periodic features. Herein, the radicals generated in Belousov-Zhabotinsky (BZ) oscillator was used to initiate RAFT (reversible additionfragmentation chain transfer) polymerization of 2-methoxyethyl acrylate (MEA) extending from hydrophilic poly(ethylene glycol) chain transfer agent (PEG-CTA) to give amphiphilic block copolymer, which self-assembled into collective objects with a size ranging from sub-micron to micron. Small-to-giant polymer vesicles could be generated using the above-mentioned BZ-PISA technology, the encapsulation of active BZ recipe into the vesicles also endorses the vesicles with growing features with potential for drug delivery and biomedical applications.

Keywords: chemical oscillation, PISA, polymeric vesicles, self-assembly, biomimetics

Chemical oscillatory reactions have been developed to mimic the behaviors of biochemical oscillations ubiquitously existing in natural living systems, characterizing as periodic, autonomous, and continuous (Kurin-Csörgei et al., 2005; Horváth et al., 2009). Among them, the BZ reaction, firstly developed as the non-organic analog to the Krebs cycle in 1951, is the mostly studied chemical oscillators in non-linear and systems chemistry (Horváth et al., 2009; Nogueira et al., 2014; Ruiz-Mirazo et al., 2014). And due to its stable, long-lasting, and repeatable oscillation features, BZ reaction has also been employed in the development of chemical tuning machine and chemical analog of noisy "metabolism" system, to handle information (Srivastava et al., 2018). The combination of $\mathrm{BZ}$ reaction with soft matters or polymeric systems has also led to numerous applications, such as the generation of self-beating or self-oscillating gels, colloidosomes, vesicles, and polymer brushes as well as chemomechanical coupling systems (Pereira de Souza and PerezMercader, 2014; Tamate et al., 2014, 2016; Masuda et al., 2015, 2016, 2018; Ren and Perez-Mercader, 2018; Onoda et al., 2017; Hu and Pérez-Mercader, 2018).

Recent years, BZ reaction has been introduced into artificial cell models development, the autonomous self-oscillating feature of $\mathrm{BZ}$ reaction allows the oscillating $\mathrm{BZ}$ reaction encapsulated liposomes, self-assembled from amphiphilic lipid, to function independently from its surroundings (Tomasi et al., 2014). And in terms of artificial cell models, polymersomes, self-assembled from amphiphilic copolymers, has attract more attentions due to that polymersomes possess higher amphiphilic molecular weights and broader chemical choice over that of lipids (Ren and PerezMercader, 2018; Rideau et al., 2018; Guo et al., 2019). The fact that polymerization need radicals 
and $\mathrm{BZ}$ reaction produce radicals further connect $\mathrm{BZ}$ reaction and polymersomes together, especially in the context of BZ reaction coupled polymerization induced self-assembly (BZPISA) (Bastakoti and Perez-Mercader, 2017a,b; Bastakoti et al., 2018). In BZ-PISA, the radicals produced in BZ reaction are used to drive polymerization of hydrophobic monomer extending from hydrophilic macromolecular RAFT agent to give amphiphilic block copolymers, which concurrently selfassembled into micelles and finally transformed into giant vesicles along with the packing parameter change in the course of the ongoing polymerization (Israelachvili, 2011; Tomasi et al., 2014; Rideau et al., 2018; Cheng and Pérez-Mercader, 2019; Guo et al., 2019). Comparing with conventional PISA that always gives nano-sized vesicles, BZ-PISA leads to giant vesicles benefiting from the autonomous $\mathrm{BZ}$ reaction and the hypersaline environment. The encapsulation of an active oscillatory chemical reaction within the polymer vesicles also endow them with important extra functionalities, including the information handling ability mentioned above, because $\mathrm{BZ}$ reaction can be used in chemical communication (PérezMercader et al., 2017), and as a chemical turning machine (Albertsen et al., 2017). However, detailed investigation of the polymerization and self-assembly process in BZ-PISA using hydrophilic monomer that polymerized into hydrophobic block has never been documented.

Herein, the radicals generated in $\mathrm{BZ}$ reaction is used to drive the polymerization of 2-methoxyethyl acrylate (MEA), a water soluble monomer that becomes hydrophobic after polymerization, extending from hydrophilic poly(ethylene glycol) chain transfer agent (PEG-CTA) to give amphiphilic deblock copolymer, poly(ethylene glycol)-block-poly(2methoxyethyl acrylate) (PEG-b-PMEA) (Figure 1). The polymerization process of PEG-b-PMEA in BZ-PISA with different target degree of polymerizations ( $\mathrm{DP}_{\text {target }} \mathrm{s}$ ) for MEA of 200, 400, and 600 are studied in detail. And the concurrent self-assembly process the morphologies of different samples at different time is also well investigated.

In pure BZ oscillatory reaction (Supplementary Figure S1), the $\mathrm{Ru}$ catalyst oscillating between $\mathrm{Ru}^{2+}$ and $\mathrm{Ru}^{3+}$, resulting in the redox potential oscillation in between $\sim 860$ and $\sim 1050 \mathrm{mV}$, with an average period of $\sim 100 \mathrm{~s}$ and an average oscillation amplitude of $\sim 160 \mathrm{mV}$. In the BZ-PISA experiments, the mixed solution of PEG-CTA and MEA monomer are fed to the $\mathrm{BZ}$ solution, and the reaction mixture is stirred at $200 \mathrm{rpm}$ (Figure 1). Foil film protection is applied to avoid any visible light-induced oxidation/reduction of Ru catalyst, which is also a radical formation process that was used to generate giant vesicles using photo PISA (Albertsen et al., 2017; Ren and PerezMercader, 2018). In the case of BZ-PISA for PEG-b-PMEA $\left(\mathrm{DP}_{\text {target }}=200\right)$, there is a long induction time of $\sim 70 \mathrm{~min}$ (Figure 2A), implying that the consumption of radicals by polymerization temporarily suspended some critical steps in the oscillation cycle documented in previous literature (Bastakoti and Perez-Mercader, 2017a,b), thus affected the oscillatory regime of $\mathrm{BZ}$ reaction. After that, the oscillation is restored, with an average period of $102 \mathrm{~s}$ and a smaller amplitude of $132 \mathrm{mV}$. For BZ-PISA of PEG-b-PMA with a $\mathrm{DP}_{\text {target }}$ of 200,

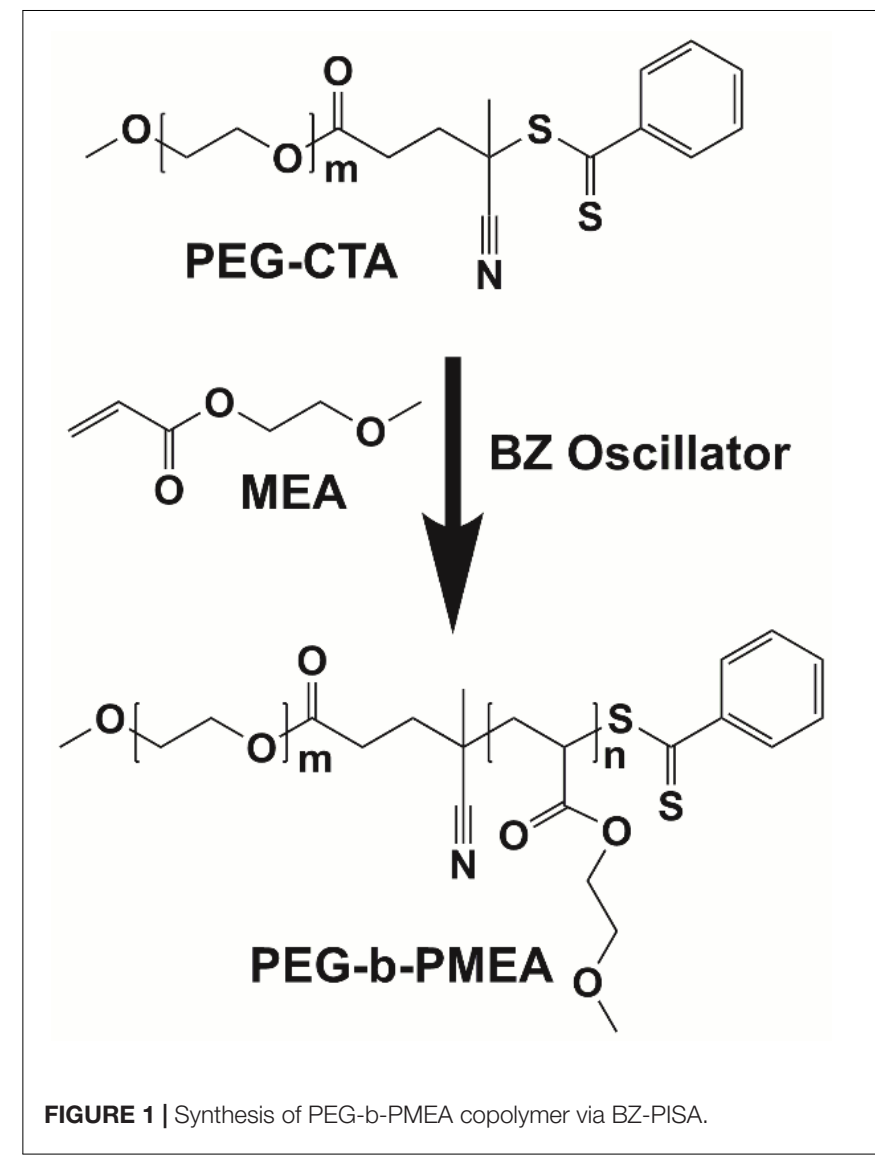

the monomer conversion increased with reaction time increase, the final monomer conversion at $120 \mathrm{~min}$ was determined to be $\sim 90 \%$ (Figure 2B). Similarly, the final monomer conversions at 120 min for BZ-PISA of PEG-b-PMEA 400 and PEG-b-PMEA 600 were all higher than $90 \%$ (Figure 3). The oscillation of BZPISA for PEG-b-PMEA exhibited different features compared with the oscillation in pure BZ. As shown in Figure 2C (lower panel), in the oscillatory region, the minimum potential of each oscillation period kept increasing from $\sim 690$ to $\sim 775 \mathrm{mV}$. While, in pure BZ oscillatory reaction (Figure 3 ), the minimum potential of each period kept decreasing from $\sim 1000$ to $\sim 875 \mathrm{mV}$ (Figure 2C, topper panel). In the cases of BZ-PISA using other monomers, including butyl acrylate (BA) (Bastakoti and PerezMercader, 2017a), acrylonitrile (Bastakoti and Perez-Mercader, 2017b), and ethyl acrylate (Bastakoti et al., 2018), the minimum potential of each period also exhibited deceasing trends. This unique feature for BZ-PISA of PEG-b-PMEA might relate to the special chemical structure of MEA, which contains an ethylene glycol unit and an ether group on the side chain, very similar chemical structure with that of PEG. The ${ }^{1} \mathrm{H}-\mathrm{NMR}$ spectra of the purified PEG-b-PMEA polymers with different $\mathrm{DP}_{\text {target }} \mathrm{S}$ and PEG, PEG-CTA are shown in Figure 2D. With the PEG peak (a in Figure 2D) being kept nearly the same, the integration areas from the peaks of PMEA ( $b$ to $f$ in Figure 2D, the ${ }^{1} \mathrm{H}$-NMR spectrum of MEA monomer is presented in Supplementary Figure S2 for comparison) became bigger and 


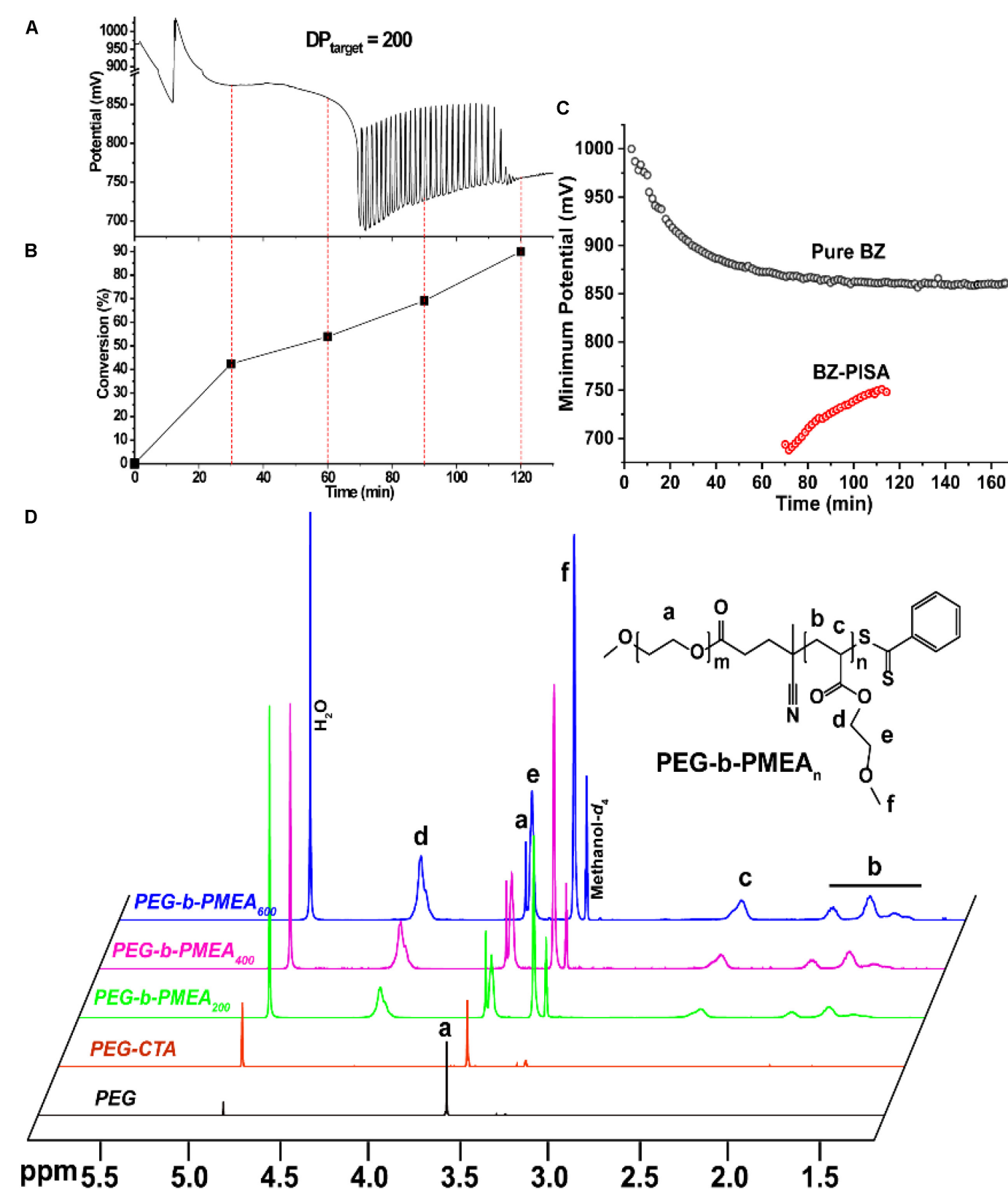

FIGURE 2 | Redox potential oscillation curve (A) and monomer conversions (B) in the course of BZ-PISA for PEG-b-PMEA with a DP target of 200. (C) Change of the minimum potentials of every periods for pure BZ reaction and BZ-PISA of PEG-b-PMA with a DP target of 200. (D) ${ }^{1} \mathrm{H}-\mathrm{NMR}$ spectra of PEG, PEG-CTA and the purified final products of BZ-PISA for PEG-b-PMEA ( $\mathrm{DP}_{\text {target }}=200,400$, and 600) after $2 \mathrm{~h}$ reaction.

bigger in the spectra of PEG-PMEA 200 , PEG-PMEA400, to PEG$\mathrm{PMEA}_{600}$, indicating the successful introduction of more MEA units into the block copolymers when the feeding ratios of MEA to PEG increased. For BZ-PISA of PEG-b-PMEA 600 , along with reaction time increasing, the increase of the areas of PMEA peaks in the ${ }^{1} \mathrm{H}-\mathrm{NMR}$ spectra can be clearly observed in Figure 4. These results indicate the decent controllability of BZ-PISA in terms of polymerization and the mutual effect between polymerization and oscillation, which was not detailed documented in previous literatures.
The self-assembly of the obtained amphiphilic block copolymers concurrent with BZ-initiated RAFT polymerization was studied by DLS and TEM measurements (Figure 5). To reflect the real situation and eliminate the reaction time during sample transport, the DLS tests were conduct on site of BZ-PISA reaction. Since MEA is water soluble, just after mixing MEA monomer with $\mathrm{BZ}$ recipe, the solution was totally transparent. Along with the dispersion radical polymerization, the solution became more and more turbid. As shown in Figure 5A1, For BZPISA of PEG-b-PMEA with a $\mathrm{DP}_{\text {target }}=200$, the hydrodynamic 


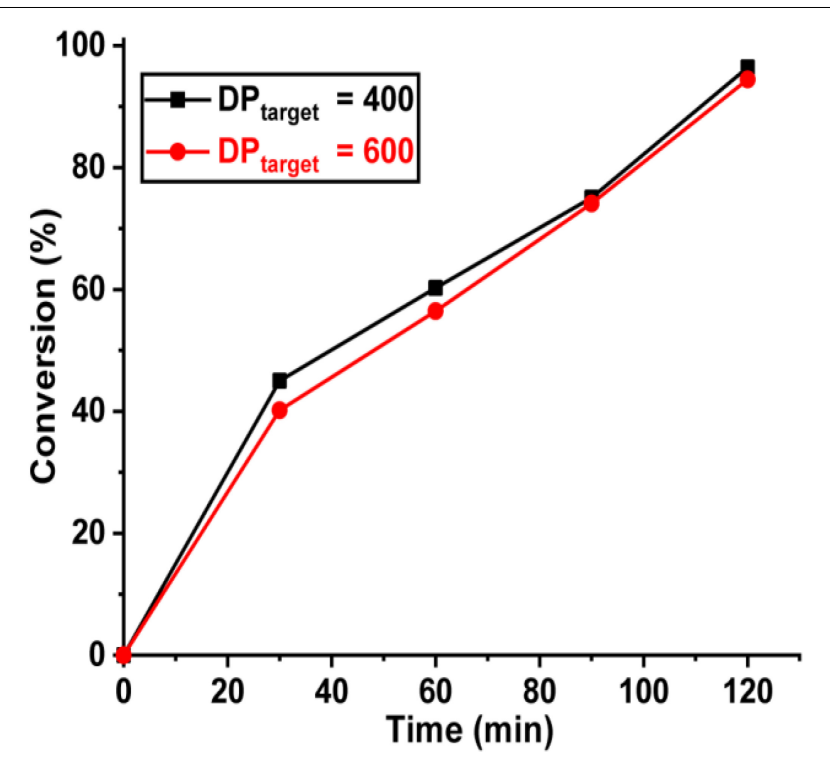

FIGURE 3 | Monomer conversions of BZ-PISA for PEG-b-PMEA with a $\mathrm{DP}_{\text {target }}$ of 400 and 600 at different time points.

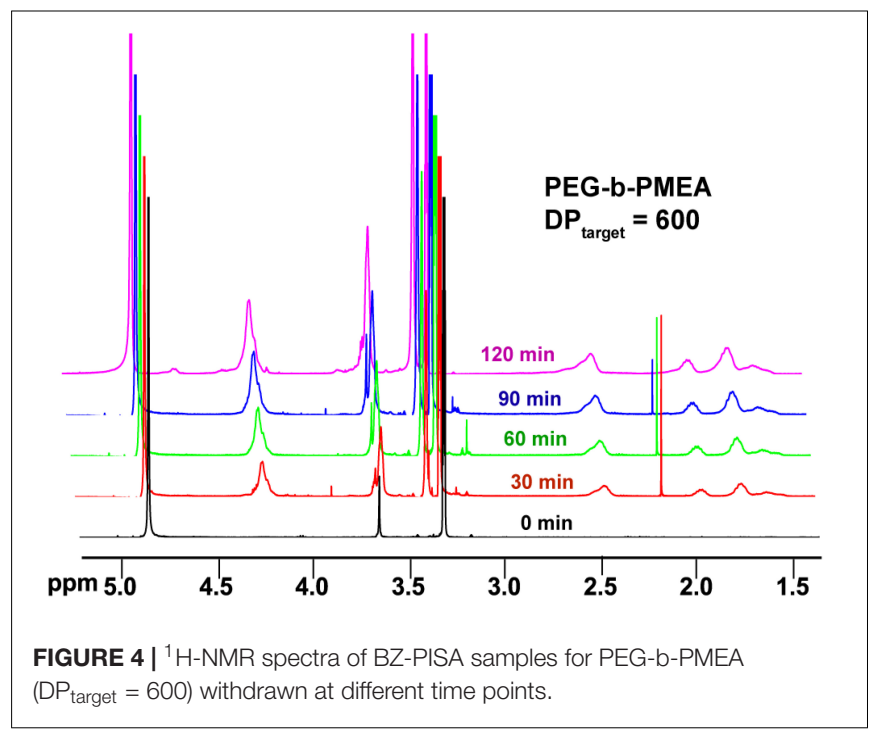

diameters of the self-assembled structures increased from $267 \mathrm{~nm}$ at $30 \mathrm{~min}$ to $491 \mathrm{~nm}$ at $120 \mathrm{~min}$. Vesicular structures are observed from the TEM images of the 60-min sample, and vesicle budding and division can be clearly seen (Figure 5A2 and Supplementary Figure S3), which is further proved by the show up of ellipse and pear-shaped structures in the enlarged TEM images (Supplementary Figure S3). For BZ-PISA of PEG-b-PMEA with a $\mathrm{DP}_{\text {target }}=400$, similar hydrodynamic diameter increase but at a larger scale, from $520 \mathrm{~nm}$ at $30 \mathrm{~min}$ to $904 \mathrm{~nm}$ at $120 \mathrm{~min}$, was also observed (Figure 5B1), and bigger vesicles than that of $\mathrm{DP}_{\text {target }}=200$ sample at $60 \mathrm{~min}$ were obtained after $60 \mathrm{~min}$ reaction (Figure 5B2). While, when $\mathrm{DP}_{\text {target }}=600$, the hydrodynamic diameters of the selfassembled structures kept decreasing from $3.19 \mu \mathrm{m}$ at $30 \mathrm{~min}$ to $2.01 \mu \mathrm{m}$ at $120 \mathrm{~min}$ (Figure 5C1). In our experiment, the molar amount of PEG-CTA was kept unchanged, increasing of $\mathrm{DP}_{\text {target }}$ means the increase of monomer content in aqueous solution. At higher monomer content, in the beginning of BZ-PISA reaction, MEA molecules might exist as droplets which were stabilized by the synthesized amphiphilic PEG-b-PMEA polymers, or the monomers were encapsulated in the inner cavity of the self-assembled polymersomes. Thus along with the consumption of monomer by polymerization, the sizes of the self-assembled structures kept decreasing. Polymer vesicles with bigger diameters were observed for the BZ-PISA of PEG-b-PMEA 600 sample at 60 min (Figure 5C2).

To further confirm the generation of giant vesicles through one-pot BZ-PISA, cryo-SEM study on the final product at 120 min of the PEG-b-PMEA with a $\mathrm{DP}_{\text {target }}=600$ was conducted, the results are shown in Figure 6. Cryo-SEM can reflect the real state of the self-assembled structures by freezing the aqueous sample quickly in frozen liquid nitrogen (pretreated under vacuum) to eliminate the formation of ice crystals, and with the help of freeze-fracture, the cross sections and possibly inner features of the self-assembled structures could be captured. From Figure 6, freeze-fractured hollow structures with a diameter $\sim 2 \mu \mathrm{m}$ could be observed, the cross sections of the vesicle membranes can also be clearly seen. Deformed and collapsed vesicle membranes, which can be clearly seen in Figures 6C,D, reflect the soft feature of the polymersomes. The thickness of vesicle membrane is measured to be $\sim 30 \mathrm{~nm}$ (Figure 6D), which is in the range of $5-50 \mathrm{~nm}$, the reported thickness of the bilayer of polymersomes (Rideau et al., 2018). The cryo-SEM results further confirm the formation of polymer vesicles in BZ-PISA of PEG-b-PMEA.

In conclusion, using the radicals generated in BelousovZhabotinsky (BZ) oscillator as initiator, a water soluble monomer, MEA, which became hydrophobic after polymerization, was polymerized extending from the active end of PEG-CTA via RAFT polymerization. The obtained amphiphilic block copolymer was concurrently self-assembled into sub-micron to micron sized collective objects. By adjusting the feeding ratio of MEA monomer to PEG-CTA from 200, 400, to 600, through one-pot BZ-mediated polymerization induced selfassembly (BZ-PISA), polymer vesicles with sizes ranging from $100 \mathrm{~nm}$ to $\sim 2.0 \mu \mathrm{m}$ were autonomously generated from simple non-amphiphilic building blocks. The encapsulation of active $\mathrm{BZ}$ recipe into polymeric giant vesicles endorses them with additional functionalities, including information handling capability, brings closer to the development of functional microreactor as artificial living system.

\section{EXPERIMENTAL PART}

\section{Materials}

Poly(ethylene glycol) 4-cyano-4(phenylcarbonothioylthio) pentanoate (PEG-CTA) was synthesized according to the literature, via the esterification reaction between methoxy poly(ethylene glycol) (mPEG, molecular weight $=1900 \mathrm{Da}$, Fluka), 4-cyano-4-(thiobenzoylthio) pentanoic acid (CTA, 

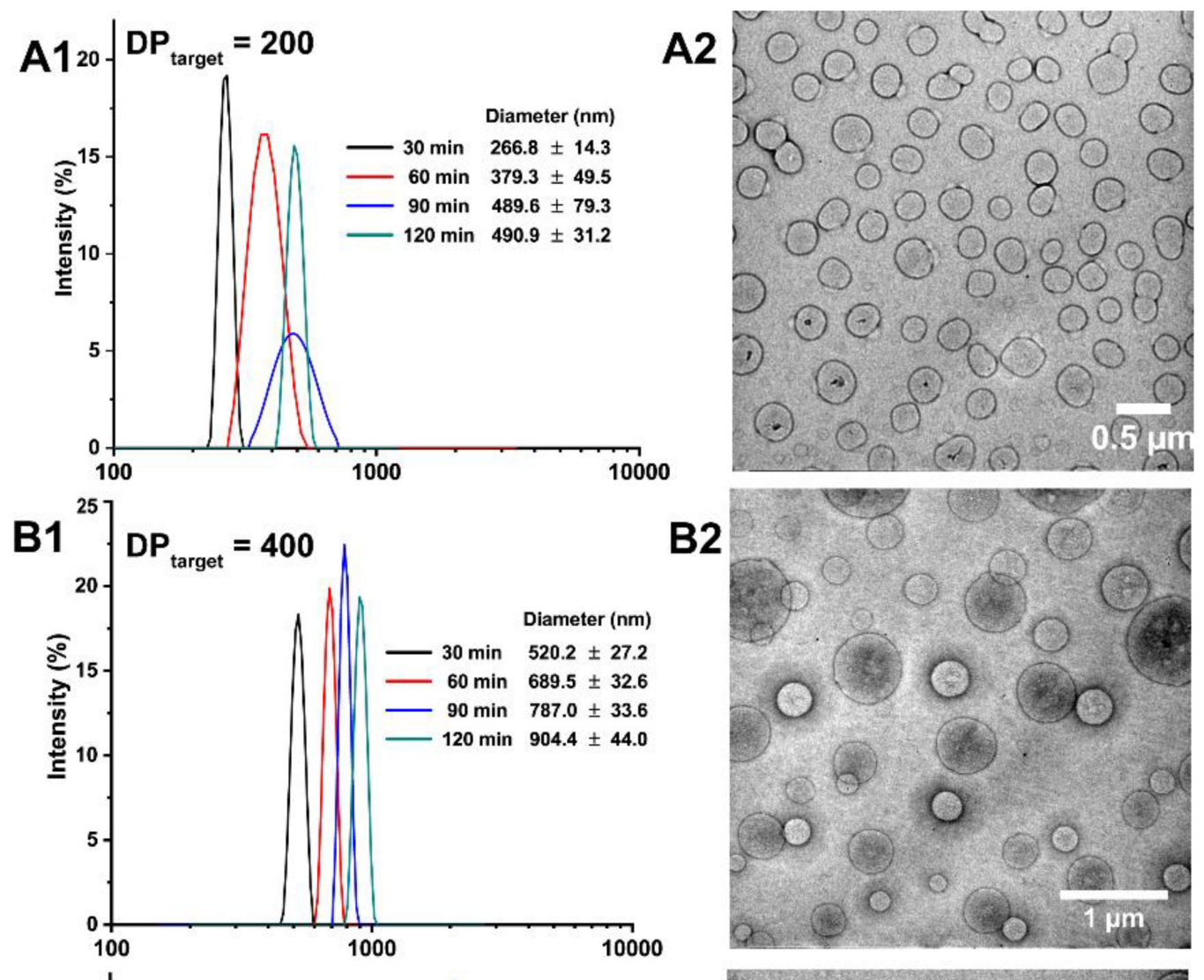

B2
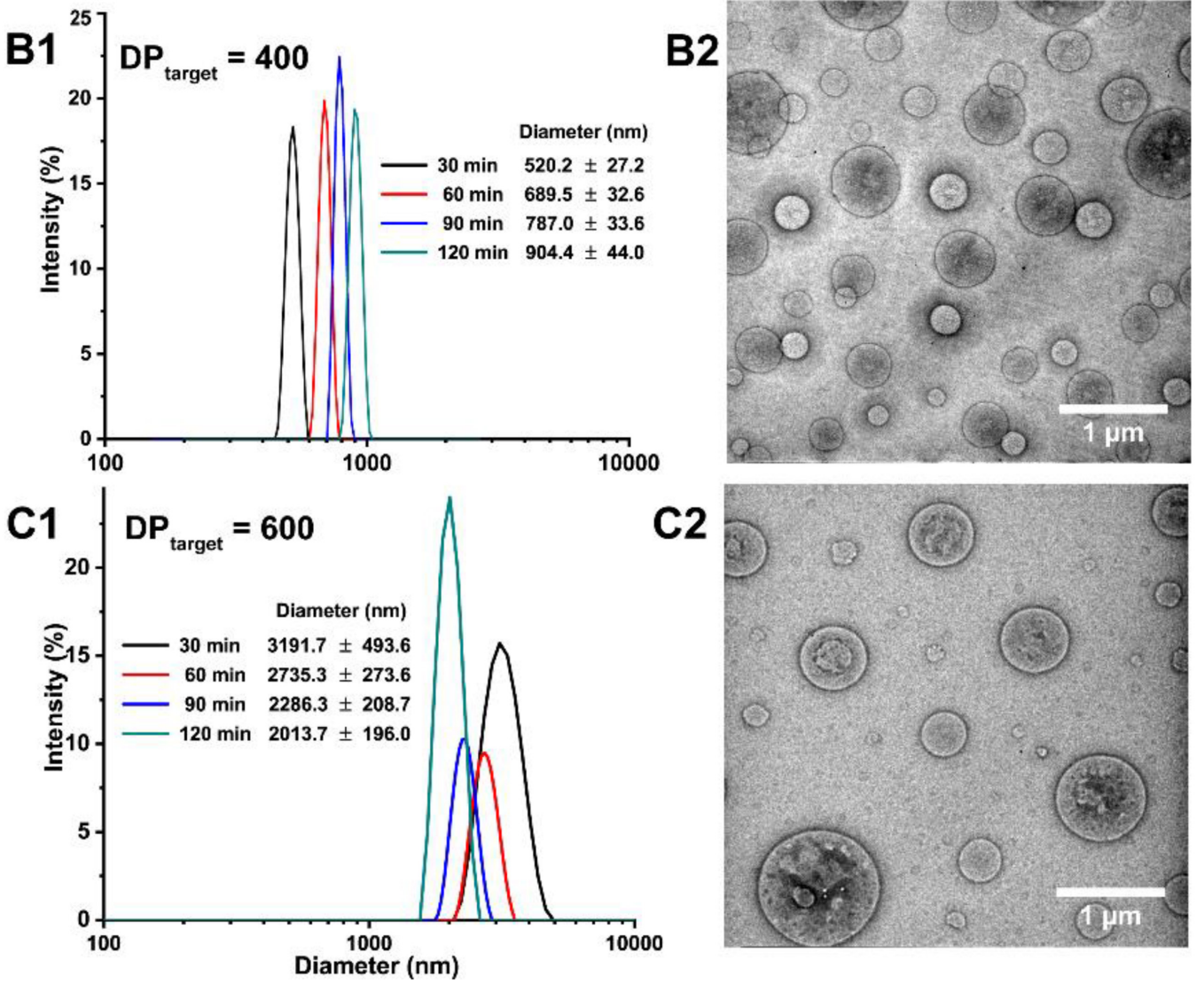

FIGURE 5 | Hydrodynamic diameters of the self-assembled structures of BZ-PISA for PEG-b-PMEA at different time points in situ monitored by DLS and corresponding TEM images of the 60-min samples: (A1,A2) DP target $=200 ;(\mathbf{B 1 , B 2}) \mathrm{DP}_{\text {target }}=400 ; \mathbf{( C 1 , \mathbf { C } 2 )} \mathbf{D P}_{\text {target }}=600$.

Strem) with the help of $\mathrm{N}, \mathrm{N}^{\prime}$-dicylohexylcarbodiimide (DCC, Sigma-Aldrich) and N,N'-dimethylamino -pyridine (DMAP, Alfa Aesar) (Szymanski and Perez-Mercader, 2016). Methanol- $d_{4}$ for ${ }^{1} \mathrm{H}-\mathrm{NMR}$ tests, was purchased from Cambridge Isotope Laboratories, Inc., 2-Methoxyethyl acrylate (MEA, SigmaAldrich), sodium hydroxide ( $\mathrm{NaOH}$, Sigma-Aldrich), sodium bromate $\left(\mathrm{NaBrO}_{3}\right.$, Sigma-Aldrich), malonic acid (MA, SigmaAldrich), tris(2,2'-bipyridyl) dichlororuthenium(II) hexahydrate ( $\mathrm{Ru}(\text { bpy })_{2} \mathrm{Cl}_{3}$, Sigma-Aldrich), sulfuric acid $\left(\mathrm{H}_{2} \mathrm{SO}_{4}, 10\right.$ Normal, Ricca Chemical Company), anhydrous dichloromethane (DCM,
Sigma-Aldrich), N,N-dimethylformamide (DMF, HPLC grade, VWR), and lithium bromide (LiBr, anhydrous, 99.99\%, VWR) were used without further purification.

\section{General Measurements}

The redox potential change of the reaction system during polymerization was monitored by a Benchtop $\mathrm{pH} / \mathrm{mV}$ Meter (Sper Scientific Direct) equipped with a MI-800 Micro-ORP Electrode (Microelectrodes Inc.), redox potential values were collected once per second. The hydrodynamic size of samples 


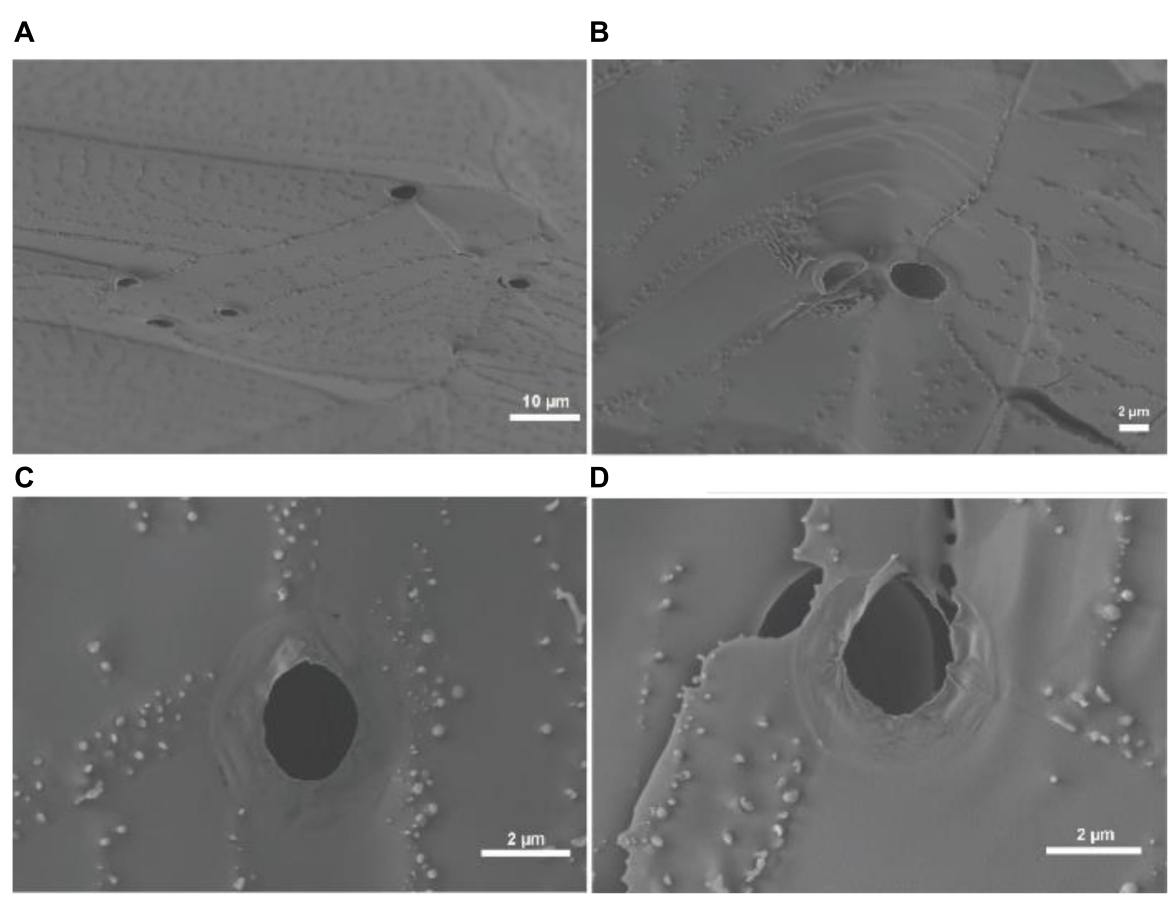

FIGURE 6 | Cryo-SEM images of the self-assembled structures of BZ-PISA for PEG-b-PMEA (DPtarget $=600)$ sampled at 120 min.

collected at different time points was measured by dynamic light scattering (DLS) analysis using a Delsa Nano C particle size and zeta potential analyzer (Beckman Coulter, Inc.). ${ }^{1} \mathrm{H}-\mathrm{NMR}$ spectra of monomer and polymers were recorded on a $500 \mathrm{MHz}$ Varian Unity/Inova 500B spectrometer using methanol- $d_{4}$ as the solvent. Morphology of the self-assembled structures was observed under transmission electron microscope (TEM, FEI Tecnai Cryo-Bio 200KV FEG TEM), field scanning electron microscope (FESEM, Supra 55VP) and/or Scanning electron cryomicroscopy (CryoSEM) [Zeiss NVision 40 focus ion beam scanning electron microscope (FIB-SEM)].

\section{Method}

The BZ coupled PISA (BZ-PISA) process is briefly described here. $17.3 \mathrm{mg}$ PEG-CTA ( $8 \mu \mathrm{mol}$ ) was dissolved in $15.465 \mathrm{~mL}$ ultrapure water. $1 \mathrm{~mL}$ malonic acid (MA, $0.6 \mathrm{M}), 2 \mathrm{~mL}$ sodium bromate $\left(\mathrm{NaBrO}_{3}, 1.0 \mathrm{M}\right), 1.2 \mathrm{~mL} \mathrm{H}_{2} \mathrm{SO}_{4}(5.0 \mathrm{M}), 206 \mu \mathrm{L}$ MEA monomers [1800 $\mu \mathrm{mol}$, target degree of polymerization $\left.\left(\mathrm{DP}_{\text {target }}\right)=200\right]$, and $0.235 \mathrm{~mL} \mathrm{Ru}(\text { bpy })_{3} \mathrm{Cl}_{2}(8.5 \mathrm{mM})$ were added in sequence and stirred at $200 \mathrm{rpm}$ at room temperature. The redox potential change of the reaction system was monitored just after the addition of all reactants. An aluminum foil was applied to protect the reaction vessel from room light. At pre-set time points $(0,30$, 60,90 , and $120 \mathrm{~min}$ ), $60 \mu \mathrm{L}$ reaction mixture was sampled out, neutralized with proper amount of $\mathrm{NaOH}$, freeze-dried to remove unreacted monomer and then dissolved in $540 \mu \mathrm{L}$ methanol- $d_{4}$ for ${ }^{1} \mathrm{H}-\mathrm{NMR}$ measurement. Fresh samples collected at 30, 60, 90, and $120 \mathrm{~min}$ were used for DLS analysis immediately. The morphology of the self-assembled structures of samples collected at $30,60,90$, and $120 \mathrm{~min}$ was observed by TEM, SEM and/or
Cryo-SEM. After $2 \mathrm{~h}$ reaction, the reaction mixture was collected, neutralized and then dialyzed against deionized water using a dialysis tubing with a molecular cut-off of 1000 Da for 3 days. Water was changed every $2 \mathrm{~h}$ in the beginning $6 \mathrm{~h}$ and every the other day after.

BZ-PISA of PEG-b-PMEA with a $\mathrm{DP}_{\text {target }}=400$ and 600 were also conducted. The amount of monomer was kept unchanged, but the amount of PEG-CTA was adjusted accordingly to obtain a $\mathrm{DP}_{\text {target }}=400$ or 600 . Pure $\mathrm{BZ}$ reaction was also conducted using the same recipe without adding PEGCTA and monomer.

\section{DATA AVAILABILITY STATEMENT}

The raw data supporting the conclusions of this article will be made available by the authors, without undue reservation.

\section{AUTHOR CONTRIBUTIONS}

ZL drafted the manuscript. JG conducted the experiments.

\section{SUPPLEMENTARY MATERIAL}

The Supplementary Material for this article can be found online at: https://www.frontiersin.org/articles/10.3389/fbioe.2020. 01018/full\#supplementary-material 


\section{REFERENCES}

Albertsen, A. N., Szymański, J. K., and Pérez-Mercader, J. (2017). Emergent properties of giant vesicles formed by a polymerization-induced self-assembly (PISA) reaction. Sci. Rep. 7:41534. doi: 10.1038/srep41534

Bastakoti, B., Guragain, S., and Pérez-Mercader, J. (2018). Direct synthesis of hundred nanometer and beyond scale polymer vesicles using chemical oscillations. Chem. Eur. J. 24, 10621-10624. doi: 10.1002/chem.201801633

Bastakoti, B. P., and Perez-Mercader, J. (2017a). Autonomous ex novo chemical assembly with blebbing and division of functional polymer vesicles from a "homogeneous mixture". Adv. Mater. 29:1704368. doi: 10.1002/adma. 201704368

Bastakoti, B. P., and Perez-Mercader, J. (2017b). Facile one-pot synthesis of functional giant polymeric vesicles controlled by oscillatory chemistry. Angew. Chem. Int. Ed. Engl. 56, 12086-12091. doi: 10.1002/anie.201703816

Cheng, G., and Pérez-Mercader, J. (2019). Polymerization-induced self-assembly for artificial biology: opportunities and challenges. Macromol. Rapid Commun. 40:1800513. doi: $10.1002 / \mathrm{marc} / 201800513$

Guo, J., Poros-Tarcali, E., and Perez-Mercader, J. (2019). Evolving Polymersomes autonomously generated and regulated by a semibatch $\mathrm{pH}$ oscillator. Chem. Commun. 55, 9383-9386. doi: 10.1039/c9cc03486b

Horváth, J., Szalai, I., and De Kepper, P. (2009). An experimental design method leading to chemical turing patterns. Science 324, 772-775. doi: 10.1126/science. 1169973

Hu, Y., and Pérez-Mercader, J. (2018). Microfluidics assisted synthesis of crosslinked colloidosomes with multisensitive behaviors: a potential platform for photo memory device and blue light-triggered release vehicle. ACS Appl. Nano Mater. 1, 3346-3354. doi: 10.1021/acsanm.8b00554

Israelachvili, J. N. (2011). Intermolecular and Surface Forces, 3rd Edn. New York, NY: Academic Press/Elsevier.

Kurin-Csörgei, K., Epstein, I. R., and Orbán, M. (2005). Systematic design of chemical oscillators using complexation and precipitation equilibria. Nature 433, 139-142. doi: 10.1038/nature03214

Masuda, T., Akimoto, A. M., Nagase, K., Okano, T., and Yoshida, R. (2016). Artificial cilia as autonomous nanoactuators: Design of a gradient selfoscillating polymer brush with controlled unidirectional motion. Sci. Adv. 2:e1600902. doi: 10.1126/sciadv.1600902

Masuda, T., Terasaki, A., Akimoto, A. M., Nagase, K., Okano, T., and Yoshida, R. (2015). Control of swelling-deswelling behavior of a self-oscillating gel by designing the chemical structure. RSC Adv. 5:5781. doi: 10.1039/C4RA10675J

Masuda, T., Ueki, T., Tamate, R., Matsukawa, K., and Yoshida, R. (2018). Chemomechanical motion of a self-oscillating gel in a protic ionic liquid. Angew. Chem. Int. Ed. Engl. 57, 16693-16697. doi: 10.1002/anie.201809413

Nogueira, P. A., Batista, B. C., Faria, R. B., and Varela, H. (2014). The effect of temperature on the dynamics of a homogeneous oscillatory system operated in batch and under flow. RSC Adv. 4, 30412-30421. doi: 10.1039/C4RA03539A
Onoda, M., Ueki, T., Tamate, R., Shibayama, M., and Yoshida, R. (2017). Amoebalike self-oscillating polymeric fluids with autonomous sol-gel transition. Nat. Commun. 8:15862. doi: 10.1038/ncomms15862

Pereira de Souza, T., and Perez-Mercader, J. (2014). Entrapment in giant polymersomes of an inorganic oscillatory chemical reaction and resulting chemo-mechanical coupling. Chem. Commun. 50, 8970-8973. doi: 10.1039/ c4cc02321h

Pérez-Mercader, J., Dueñas-Diez, M., and Case, D. (2017). Chemically-operated turing machine. US Patent 9582771:B2.

Ren, K., and Perez-Mercader, J. (2018). Light-induced evolution of microaggregates: transformation to vesicles, cyclic growth and collapse and vesicle fusion. Polym. Chem. 9, 3594-3599. doi: 10.1039/C8PY00546J

Rideau, E., Dimova, R., Schwille, P., Wurm, F. R., and Landfester, K. (2018). Liposomes and polymersomes: A comparative review towards cell mimicking. Chem. Soc. Rev. 47, 8572-8610. doi: 10.1039/C8CS00162F

Ruiz-Mirazo, K., Briones, C., and de la Escosura, A. (2014). Prebiotic systems chemistry: new perspectives for the origins of life. Chem. Rev. 114, 285-366. doi: $10.1021 / \mathrm{cr} 2004844$

Srivastava, R., Dueñas-Díez, M., and Pérez-Mercader, J. (2018). Feed rate noise modulates autocatalysis and shapes the oscillations of the BelousovZhabotinsky reaction in a continuous stirred tank reactor. React. Chem. Eng. 3, 216-226. doi: 10.1039/C7RE00196G

Szymanski, J. K., and Perez-Mercader, J. (2016). Direct optical observations of vesicular self-assembly in large-scale polymeric structures during photocontrolled biphasic polymerization. Polym. Chem. 7, 7211-7215. doi: 10.1039/C6PY01497F

Tamate, R., Ueki, T., Shibayama, M., and Yoshida, R. (2014). Self-oscillating vesicles: Spontaneous cyclic structural changes of synthetic diblock copolymers. Angew. Chem. Int. Ed. Engl. 53, 11248-11252. doi: 10.1002/anie.201406953

Tamate, R., Ueki, T., and Yoshida, R. (2016). Evolved colloidosomes undergoing cell-like autonomous shape oscillations with buckling. Angew. Chem. Int. Ed. Engl. 55, 5179-5183. doi: 10.1002/anie.201511871

Tomasi, R., Noel, J.-M., Zenati, A., Ristori, S., Rossi, F., Cabuil, V., et al. (2014). Chemical communication between liposomes encapsulating a chemical oscillatory reaction. Chem. Sci. 5, 1854-1859. doi: 10.1039/C3SC53227E

Conflict of Interest: The authors declare that the research was conducted in the absence of any commercial or financial relationships that could be construed as a potential conflict of interest.

Copyright (C) $2020 \mathrm{Lu}$ and Guo. This is an open-access article distributed under the terms of the Creative Commons Attribution License (CC BY). The use, distribution or reproduction in other forums is permitted, provided the original author(s) and the copyright owner(s) are credited and that the original publication in this journal is cited, in accordance with accepted academic practice. No use, distribution or reproduction is permitted which does not comply with these terms. 